ANNALES

POLONICI MATHEMATICI

$91.1(2007)$

\title{
Formule d'homotopie pour un domaine à bord $(q+k)$-concave d'une variété CR générique et $q$-concave. Applications
}

\author{
par Salomon Sambou et Bocar Touré (Dakar)
}

\begin{abstract}
We give a homotopy formula for the $\bar{\partial}_{b}$ operator on a domain with $(q+k)$ concave boundary. As a consequence we show that the dimension of the $\bar{\partial}_{b}$-cohomology groups for some CR manifolds $q$-concave at infinity is finite.
\end{abstract}

1. Introduction. Ce travail s'inscrit dans le cadre général de l'étude de l'équation de Cauchy-Riemann tangentielle. Plus précisement nous résolvons le $\bar{\partial}_{b}$ dans un domaine $\omega_{\tau}$ d'une sous-variété $\mathrm{CR}$ générique de codimension $k$ de $\mathbb{C}^{n}$ et $q$-concave. Nous définissons $\omega_{\tau}$ comme dans le théorème 4.3 de [5] : $\omega_{\tau}=\left\{z \in M|| z-z_{0} \mid<\tau\right.$ et $\left.\varphi(z)<\varphi\left(z_{0}\right)\right\}, \varphi$ étant une fonction $(q+k)$-concave sur un voisinage d'un point $z_{0}$ de $M$ et $\tau$ un réel strictement positif assez petit pour que $\omega_{\tau} \subset \subset M$ (voir sous-section 3.1).

Notre résultat principal est le théorème suivant où $C_{n, r}\left(\omega_{\tau}\right)$ désigne l'espace des $(n, r)$-formes différentielles continues sur $\omega_{\tau}$ :

Théorème 1.1. Soit $M$ une sous-variété $C R$ générique de $\mathbb{C}^{n}$ de codimension réelle $k$ de classe $C^{3}$ et $q$-concave $(1 \leq q \leq(n-k) / 2)$. Pour tout $z_{0}$ dans $M$ et tout domaine $\omega_{\tau}$ de $M$ défini comme précédemment il existe des opérateurs intégraux $T_{r}$ de $C_{n, r}\left(\bar{\omega}_{\tau}\right)$ dans $C_{n, r-1}^{1 / 2-\varepsilon}\left(\omega_{\tau^{\prime}}\right), 0<\tau^{\prime}<\tau$, tels que pour toute forme différentielle $f$ de bidegré $(n, r)$ et de classe $C^{1}$ sur $\bar{\omega}_{\tau}$ on $a$, sur $\omega_{\tau^{\prime}}$ :

(i) $f=\bar{\partial}_{b} T_{r} f+T_{r+1} \bar{\partial} f$ si $1 \leq r \leq q-3$,

(ii) $f=\bar{\partial}_{b} T_{r} f$ si $r=q-2$ et $\bar{\partial} f=0$.

Le théorème 1.1 est l'équivalent en codimension supérieure du théoréme 4.3 de [5] qui traite des hypersurfaces réelles de $\mathbb{C}^{n}, q$-concaves-convexes avec des estimations jusqu'au bord. Par ailleurs, utilisant des estimations

2000 Mathematics Subject Classification: 32V20, 32F10.

Key words and phrases: homotopy formula, CR manifold, $q$-concave, $q$-concave at infinity. 
holdériennes jusqu'au bord faites dans [6], nous dérivons du théorème 1.1 le résultat suivant :

THÉORÈme 1.2. Les opérateurs $T_{r}$ sont continus de $C_{n, r}^{0}\left(\bar{\omega}_{\tau}\right)$ dans $C_{n, r-1}^{1 / 2-\varepsilon}\left(\bar{\omega}_{\tau^{\prime}}\right)$ pour tous $0<\varepsilon<1 / 2$ et $1 \leq r \leq q-2$.

Enfin, moyennant une hypothèse supplémentaire de $q$-concavité à l'infini sur $M$, on déduit du théorème 1.1 une version (en codimension quelconque) dans le cas "concave" de quelques théorèmes de finitude et d'invariance de groupes de cohomologie obtenus dans [5]. Une version $C^{\infty}$ des résultats de même nature a été obtenue par Ricard dans [7], à partir des solutions locales $\mathrm{du} \bar{\partial}_{b}$ qui ne proviennent pas d'une formule d'homotopie.

2. Généralités. Soit $M$ une sous-variété réelle de classe $C^{3}$ de $\mathbb{C}^{n}$. Soit $k$ la codimension réelle de $M(1 \leq k \leq n)$. Si $z \in M$, on note par $T_{z}^{\mathbb{C}} M$ l'espace tangent complexe à $M$ en $z$.

Définition 2.1. $M$ est dite $C R$ (Cauchy-Riemann) si $\operatorname{dim}_{\mathbb{C}} T_{z}^{\mathbb{C}} M$ est indépendante de $z \in M$.

Définition 2.2. $M$ est $C R$ générique si $\operatorname{dim}_{\mathbb{C}} T_{z}^{\mathbb{C}} M=n-k$ pour tout $z \in M$.

Soient $U \subset \mathbb{C}^{n}$ un ouvert de $\mathbb{C}^{n}$ et $\widehat{\varrho}_{1}, \ldots, \widehat{\varrho}_{k}$ des fonctions de classe $C^{2}$ sur $U$ telles que

$$
M \cap U=\left\{z \in U \mid \widehat{\varrho}_{1}(z)=\cdots=\widehat{\varrho}_{k}(z)=0\right\} \quad \text { avec } \quad d \widehat{\varrho}_{1} \wedge \cdots \wedge d \widehat{\varrho}_{k} \neq 0 .
$$

La variété $M$ est alors CR générique si et seulement si $\bar{\partial} \widehat{\varrho}_{1} \wedge \cdots \wedge \bar{\partial} \widehat{\varrho}_{k} \neq 0$ sur $M \cap U$.

Les fonctions $\widehat{\varrho}_{1}, \ldots, \widehat{\varrho}_{k}$ sont appelées fonctions définissantes de $M$ sur $U$.

DÉFInition 2.3. Une sous-variété réelle $M$ de $\mathbb{C}^{n}, \mathrm{CR}$ générique de codimension réelle $k$ est dite $q$-concave, $1 \leq q \leq(n-k) / 2$, si pour tout $z \in M$ et tout $x=\left(x_{1}, \ldots, x_{k}\right) \in \mathbb{R}^{k} \backslash\{0\}$ la forme de Levi, restreinte à $T_{z}^{\mathbb{C}} M$,

$$
L \widehat{\varrho}_{x}(t)=\sum_{\alpha, \beta=1}^{n} \frac{\partial^{2} \widehat{\varrho}_{x}}{\partial z^{\alpha} \partial \bar{z}^{\beta}}(z) t^{\alpha} \bar{t}^{\beta},
$$

où $\widehat{\varrho}_{x}=x_{1} \widehat{\varrho}_{1}+\cdots+x_{k} \widehat{\varrho}_{k}$, admet au moins $q$ valeurs propres strictement négatives.

DÉfinition 2.4. Soit $M$ une sous-variété CR générique de codimension réelle $k$ de $\mathbb{C}^{n}$ et $q$-concave. On dit qu'une fonction $\varphi$ définie sur $M$ est $(q+k)$-concave (respectivement $(q+k)$-convexe $)$ sur $M$ si pour tout point $p$ de $M$ et pour toute extension $\psi$ de classe $C^{2}$ de $\varphi$ (respectivement $-\varphi$ ) à un voisinage de $p$ dans $\mathbb{C}^{n}$, la forme de Levi de $\psi$ restreinte à l'espace 
tangent complexe à $M$ en $p$ possède au moins $q$ valeurs propres strictement négatives.

3. Formules locales. Nous nous proposons dans cette partie de construire des opérateurs intégraux $T_{r}$ en vue d'obtenir des formules locales permettant de résoudre le $\bar{\partial}_{b}$. Tout d'abord rappelons brièvement la construction du noyau de Barkatou-Laurent $R_{M}$.

On désigne par $\mathcal{I}$ l'ensemble des parties $I \subseteq\{ \pm 1, \cdots, \pm k\}$ tel que $|i| \neq|j|$ pour tous $i, j \in \mathcal{I}$ distincts. Si $I \in \mathcal{I}$, alors on note par $|I|$ le nombre d'éléments de $I$ et par $\triangle_{I}$ le simplexe de $\mathbb{R}^{k}$ défini par

$$
\triangle_{I}=\left\{\lambda \in \mathbb{R}^{k}: \lambda=\sum_{j=1}^{|I|} \lambda_{j} e_{j}, \lambda_{j} \geq 0, \sum_{j=1}^{|I|} \lambda_{j}=1\right\}
$$

où $\left(e_{j}\right)_{1 \leq j \leq k}$ est la base canonique de $\mathbb{R}^{k}$ avec la convention suivante $: e_{-j}=$ $-e_{j}$ pour tout $j=1, \ldots, k$. Soit $l$ un entier naturel tel que $1 \leq l \leq k$. On note par $\mathcal{I}(l)$ l'ensemble de tous les $I \in \mathcal{I}$ tels que $|I|=l$, et par $\mathcal{I}^{\prime}(l)$ l'ensemble de tous les $I \in \mathcal{I}(l)$ tels que si $I=\left(i_{1}, \ldots, i_{l}\right)$ alors $\left|i_{\nu}\right|=\nu$ pour tout $\nu=1, \ldots, l$. Enfin, si $I \in \mathcal{I}$ alors on pose

$$
\operatorname{sgn}(I)= \begin{cases}+1 & \text { si le nombre d'éléments négatifs de } I \text { est pair, } \\ -1 & \text { si le nombre d'éléments négatifs de } I \text { est impair. }\end{cases}
$$

Fixons $z_{0} \in M$. Soit $U_{z_{0}} \subset \mathbb{C}^{n}$ un voisinage de $z_{0}$. Puisque $M$ est $q$ concave, d'après le lemme 3.1.1 de [1] il existe une constante $C>0$ telle que pour $j=1, \ldots, k$ les fonctions

$$
\varrho_{j}=\widehat{\varrho}_{j}+C \sum_{\nu=1}^{k} \widehat{\varrho}_{\nu}^{2}, \quad \varrho_{-j}=-\widehat{\varrho}_{j}+C \sum_{\nu=1}^{k} \widehat{\varrho}_{\nu}^{2}
$$

possèdent la propriété suivante : pour tout $I=\left(i_{1}, \ldots, i_{|I|}\right) \in \mathcal{I}$ et tout $\lambda \in \triangle_{I}$ la forme de Levi de $\lambda_{i_{1}} \varrho_{i_{1}}+\cdots+\lambda_{i_{|I|}} \varrho_{i_{|I|}}$ en $z_{0}$ admet au moins $q+k$ valeurs propres strictement positives. Pour tout $I \in \mathcal{I}^{\prime}(k)$, on note $\mathcal{I}^{\prime}(k, *)$ l'ensemble des multi-indices $I_{*}=\left(i_{1}, \ldots, i_{k}, *\right)$, où $I=\left(i_{1}, \ldots, i_{k}\right)$. On pose $\varrho_{*}=\varrho_{1}+\cdots+\varrho_{k}$ et $\varrho_{\lambda}=\lambda_{1} \varrho_{i_{1}}+\cdots+\lambda_{k} \varrho_{i_{k}}+\lambda_{*} \varrho_{*}$ pour $\lambda=$ $\left(\lambda_{1}, \ldots, \lambda_{k}, \lambda_{*}\right) \in \triangle_{I_{*}}$.

On construit alors à l'aide de $\varrho_{\lambda}$ ainsi défini des sections de Leray $\left(\psi_{J}\right)_{J \in \mathcal{I}^{\prime}(k, *)}$ (cf. [2]) et des noyaux $K_{J}$ et $C_{J}$ associés à $\psi_{J}$.

$\operatorname{Pour}(z, \xi, \lambda) \in U_{z_{0}} \times U_{z_{0}} \times \triangle_{I_{*}}$ avec $z \neq \xi$, on pose

$$
K_{I_{*}}(z, \xi, \lambda)=\frac{(-1)^{n(n-1) / 2}}{(2 \pi i)^{n}}\left\langle\psi_{I_{*}}, d(\xi-z)\right\rangle \wedge\left\langle\left(\bar{\partial}_{\xi, z}+d_{\lambda}\right) \psi_{I_{*}}, d(\xi-z)\right\rangle^{n-1} .
$$


Pour $(z, \xi) \in U_{z_{0}} \times U_{z_{0}}$ avec $z \neq \xi$, on pose

$$
C_{I_{*}}=\int_{\lambda \in \triangle_{I_{*}}} K_{I_{*}}(z, \xi, \lambda)
$$

Le noyau de Barkatou-Laurent est alors défini par

$$
R_{M}=\sum_{I \in \mathcal{I}^{\prime}(k)} \operatorname{sgn}(I) C_{I_{*}} .
$$

Le résultat fondamental suivant est dû à Christine Laurent-Thiébaut et Moulay Y. Barkatou [2].

ThÉORÈme 3.1. Soit $\omega$ un domaine à bord $C^{1}$ tel que $\omega \subset \subset M \cap U$, et $f$ une $(n, r)$-forme de classe $C^{1}$ sur $\bar{\omega}$. Si $n-k-q+1 \leq r \leq n-k$ alors on $a$

$$
\begin{aligned}
(-1)^{(n+r)(k+1)+2 k(k+1) / 2} f(z)= & (-1)^{k} \int_{\xi \in \partial \omega} f(\xi) \wedge\left[R_{M}\right]_{n, r}(z, \xi) \\
& +(-1)^{k+1} \int_{\xi \in \omega} \bar{\partial}_{\xi} f(\xi) \wedge\left[R_{M}\right]_{n, r}(z, \xi) \\
& +\bar{\partial}_{z} \int_{\xi \in \omega} f(\xi) \wedge\left[R_{M}\right]_{n, r-1}(z, \xi) .
\end{aligned}
$$

Si $0 \leq r \leq q-1$ on $a$

$$
\begin{aligned}
(-1)^{(n+r)(k+1)+k(k+1) / 2} f(\xi) & (-1)^{k} \int_{z \in \partial \omega} f(z) \wedge\left[R_{M}\right]_{0, n-k-r-1}(z, \xi) \\
& +(-1)^{k+1} \int_{z \in \omega} \bar{\partial}_{z} f(z) \wedge\left[R_{M}\right]_{0, n-k-r-1}(z, \xi) \\
& +\bar{\partial}_{\xi} \int_{z \in \omega} f(z) \wedge\left[R_{M}\right]_{0, n-k-r}(z, \xi) .
\end{aligned}
$$

On a là une formule du type Bochner-Martinelli-Koppelman (BMK) pour variétés $\mathrm{CR}$ génériques $q$-concaves.

Pour construire nos opérateurs intégraux, nous passerons par les étapes suivantes :

(i) définition du domaine $\omega_{\tau}$ et décomposition de son bord $\partial \omega_{\tau}$ en deux parties $W_{1}$ et $W_{2}$,

(ii) transformation de l'intégrale $\int_{z \in W_{2}} f(z) \wedge\left[R_{M}\right]_{0, n-k-r-1}(z, \xi)$,

(iii) utilisation de l'opérateur de Henkin,

(iv) application du théorème 3.1.

On suppose que $M$ est une sous-variété réelle de $\mathbb{C}^{n}$, de codimension réelle $k$, CR générique et $q$-concave $(1 \leq k \leq n$ et $1 \leq q \leq(n-k) / 2)$. 
3.1. Définition de $\omega_{\tau}$ et décomposition de $\partial \omega_{\tau}$. Soit $z_{0} \in M$. On considère $\varphi$ une fonction de classe $C^{3},(q+k)$-concave sur un voisinage de $Z_{0}$ dans $M$ et telle que $d \varphi\left(z_{0}\right) \neq 0$. Posons :

$$
\begin{aligned}
U_{z_{0}}= & \left\{z \in \mathbb{C}^{n}|| z-z_{0} \mid<\tau\right\}, \quad \tau \in \mathbb{R}_{+}^{\star}, \\
\Omega_{\tau}= & \left\{z \in U_{z_{0}} \mid \psi(z)<\varphi\left(z_{0}\right)\right\} \\
& \text { où } \psi \text { est une extension de classe } C^{2} \text { de } \varphi \text { à } U_{z_{0}}, \\
\omega_{\tau}= & \Omega \cap M, \\
W_{1}= & \left\{z \in M \mid \varphi(z) \leq \varphi\left(z_{0}\right) \text { et }\left|z-z_{0}\right|=\tau\right\}, \\
W_{2}= & \left\{z \in M \mid \varphi(z)=\varphi\left(z_{0}\right) \text { et }\left|z-z_{0}\right| \leq \tau\right\} .
\end{aligned}
$$

Nous avons alors

$$
\partial \omega_{\tau}=W_{1} \cup W_{2}, \quad \partial W_{1}=\partial W_{2}=\left\{z \in M \mid \varphi(z)=\varphi\left(z_{0}\right) \text { et }\left|z-z_{0}\right|=\tau\right\} .
$$

On a donc la formule

$$
\begin{aligned}
\int_{z \in \partial \omega_{\tau}} f(z) \wedge\left[R_{M}\right]_{0, n-k-r-1}(z, \xi)= & \int_{z \in W_{1}} f(z) \wedge\left[R_{M}\right]_{0, n-k-r-1}(z, \xi) \\
& +\int_{z \in W_{2}} f(z) \wedge\left[R_{M}\right]_{0, n-k-r-1}(z, \xi) .
\end{aligned}
$$

3.2. Transformation de l'intégrale sur $W_{2}$. On a établi dans [6] la relation

$$
\bar{\partial}_{z, \xi} G_{M}(z, \xi)=(-1)^{k}\left(R_{M}(z, \xi)-S_{M}(z, \xi)\right) .
$$

Il s'ensuit que

$$
\begin{aligned}
\int_{z \in W_{2}} f(z) \wedge \bar{\partial}_{z}\left[G_{M}\right]_{0, n-k-r-2}(z, \xi) & +\int_{z \in W_{2}} f(z) \wedge \bar{\partial}_{\xi}\left[G_{M}\right]_{0, n-k-r-1}(z, \xi) \\
= & (-1)^{k} \int_{z \in W_{2}} f(z) \wedge\left[R_{M}\right]_{0, n-k-r-1}(z, \xi) \\
& +(-1)^{k+1} \int_{z \in W_{2}} f(z) \wedge\left[S_{M}\right]_{0, n-k-r-1}(z, \xi) .
\end{aligned}
$$

(Pour les définitions des noyaux $G_{M}$ et $S_{M}$, voir [6].) $W_{2}$ est dans le bord de l'ouvert $\left\{z \in M \mid-\varphi(z)<-\varphi\left(z_{0}\right)\right.$ et $\left.\left|z-z_{0}\right|<\tau\right\}$. Comme $\varphi$ est $(q+k)$-concave, $-\varphi$ est $(q+k)$-convexe. Par conséquent, les applications de Leray $\psi_{I_{*}}$ définies dans la section 2 de [8] sont $(q+k)$-holomorphes en $z$. De plus, pour $0 \leq r \leq q-2$ on a $n-k-q+1 \leq n-k-r-1$. Par suite, on a les annulations $\left[S_{M}\right]_{0, n-k-r-1}=0$ pour $n-k-r-1 \geq n-k-q+1$.

Par ailleurs on a aussi les formules suivantes :

$$
\int_{z \in W_{2}} f(z) \wedge \bar{\partial}_{\xi}\left[G_{M}\right]_{0, n-k-r-1}(z, \xi)=(-1)^{n+r+k+1} \bar{\partial}_{\xi} G_{W_{2}}^{r}(f)(\xi)
$$


et

$$
\begin{aligned}
\int_{z \in W_{2}} f(z) \wedge \bar{\partial}_{z}\left[G_{M}\right]_{0, n-k-r-2}(z, \xi)= & (-1)^{n+r+1} \bar{\partial}_{\xi} G_{W_{2}}^{r+1}\left(\partial_{z} f\right)(\xi) \\
& +(-1)^{n+r} G_{\partial W_{2}}^{r+1}(f)(\xi)
\end{aligned}
$$

où l'on a posé (momentanément)

$$
G_{V}^{r}(g)=\int_{z \in V} g(z) \wedge \bar{\partial}_{\xi}\left[G_{M}\right]_{0, n-k-r-1}(z, \cdot)
$$

avec soit $V=W_{2}$, soit $V=\partial W_{2}$.

Donc finalement on obtient

$$
\begin{aligned}
(-1)^{k} \int_{z \in W_{2}} f(z) \wedge\left[R_{M}\right]_{0, n-k-r-1}(z, \xi)= & (-1)^{n+r+k+1} \bar{\partial}_{\xi} G_{W_{2}}^{r}(f)(\xi) \\
& +(-1)^{n+r+1} G_{W_{2}}^{r+1}\left(\partial_{\xi} f\right)(\xi) \\
& +(-1)^{n+r} G_{\partial W_{2}}^{r+1}(f)(\xi) .
\end{aligned}
$$

3.3. Utilisation de l'opérateur de Henkin. Pour $U_{z_{0}}^{\prime}=\left\{z \in U_{z_{0}}|| z-z_{0} \mid\right.$ $<\tau^{\prime}$ \} avec $0<\tau^{\prime}<\tau$ on pose

$$
\begin{aligned}
& \widetilde{P}_{r} f(\xi)=(-1)^{\alpha+k} \int_{z \in W_{1}} f(z) \wedge\left[R_{M}\right]_{0, n-k-r-1}, \quad \widetilde{P}_{r} f \in C_{n, r}^{0}(\omega) . \\
& \widetilde{\widetilde{P}}_{r} f(\xi)=(-1)^{n+r+\alpha} \int_{z \in \partial W_{2}} f(z) \wedge\left[G_{M}\right]_{0, n-k-r-2}(z, \xi), \quad \widetilde{\widetilde{P}}_{r} f \in C_{n, r}^{0}(\omega),
\end{aligned}
$$

où $\alpha=(n+r)(k+1)+\frac{1}{2} k(k+1)=\alpha(r)$. Posons $P_{r} f=\widetilde{P}_{r} f+\widetilde{\widetilde{P}}_{r} f$ sur $U_{z_{0}}^{\prime}$. Pour $1 \leq r \leq q-1$, soit $L_{r}$ l'opérateur de Henkin (cf. [3]) permettant de résoudre le $\bar{\partial}$ dans la boule $U_{z_{0}}^{\prime}$ de $\mathbb{C}^{n}$. Alors $L_{r}: C_{n, r}^{0}\left(U_{z_{0}}\right) \rightarrow C_{n, r-1}^{\varepsilon}\left(U_{z_{0}}^{\prime}\right)$, $0<\varepsilon<1$, est linéaire et continue. De plus, sur $U_{z_{0}}^{\prime}$, on a

$$
\bar{\partial} L_{r} \widetilde{P}_{r} f+L_{r+1} \bar{\partial} \widetilde{P}_{r} f=\widetilde{P}_{r} f .
$$

On a aussi une relation analogue avec $\widetilde{\widetilde{P}}_{r}$. Par conséquent, $\bar{\partial} L_{r} P_{r} f+L_{r+1} \bar{\partial} P_{r} f$ $=P_{r} f$. On déduit de ce qui précède, pour $\xi \in U_{z_{0}}^{\prime}$, la formule

$$
\begin{aligned}
(-1)^{k} \int_{z \in \partial \omega_{\tau}} f(z) \wedge & {\left[R_{M}\right]_{0, n-k-r-1}(z, \xi) } \\
= & (-1)^{\alpha} \widetilde{P}_{r} f(\xi)+(-1)^{\alpha} \widetilde{\widetilde{P}}_{r} f(\xi) \\
& +(-1)^{n+r+k+1} \bar{\partial}_{\xi} \int_{z \in W_{2}} f(z) \wedge\left[G_{M}\right]_{0, n-k-r-1}(z, \xi) \\
& +(-1)^{n+r+1} \int_{z \in W_{2}} \bar{\partial} f(z) \wedge\left[G_{M}\right]_{0, n-k-r-2}(z, \xi) .
\end{aligned}
$$


3.4. Conséquence de la formule BMK pour variétés $C R$. En tenant compte de (3) dans le (2) du théorème 3.1, on a, pour $\xi \in \omega_{\tau^{\prime}}=\omega_{\tau} \cap U_{z_{0}}^{\prime}$,

$$
\begin{aligned}
f(\xi)= & \widetilde{P}_{r} f(\xi)+\widetilde{\widetilde{P}}_{r} f(\xi)+(-1)^{n+r+\alpha+k+1} \bar{\partial}_{\xi} \int_{z \in W_{2}} f(z) \wedge\left[G_{M}\right]_{0, n-k-r-1}(z, \xi) \\
& +(-1)^{n+r+\alpha+1} \int_{z \in W_{2}} \bar{\partial}_{z} f(z) \wedge\left[G_{M}\right]_{0, n-k-r-2}(z, \xi) \\
& +(-1)^{\alpha+k+1} \int_{z \in \omega_{\tau}} \bar{\partial}_{z} f(z) \wedge\left[R_{M}\right]_{0, n-k-r-1}(z, \xi) \\
& +(-1)^{\alpha} \bar{\partial}_{\xi} \int_{z \in \omega_{\tau}} f(z) \wedge\left[R_{M}\right]_{0, n-k-r}(z, \xi),
\end{aligned}
$$

ce qui entraîne encore

$$
\begin{aligned}
f(\xi)= & \left\{L_{r+1} \bar{\partial} P_{r} f(\xi)+(-1)^{n+r+\alpha+1} \int_{z \in W_{2}} \bar{\partial}_{z} f(z) \wedge\left[G_{M}\right]_{0, n-k-r-2}(z, \xi)\right. \\
& \left.+(-1)^{\alpha+k+1} \int_{z \in \omega_{\tau}} \bar{\partial}_{z} f(z) \wedge\left[R_{M}\right]_{0, n-k-r-1}(z, \xi)\right\}+\bar{\partial}_{\xi} L_{r} P_{r} f(\xi) \\
& +(-1)^{n+r+\alpha+k+1} \int_{z \in W_{2}} f(z) \wedge\left[G_{M}\right]_{0, n-k-r-1}(z, \xi) \\
& +(-1)^{\alpha} \int_{z \in \omega_{\tau}} f(z) \wedge\left[R_{M}\right]_{0, n-k-r}(z, \xi) .
\end{aligned}
$$

On a donc

$$
\begin{aligned}
f(\xi)= & \bar{\partial}_{\xi}\left\{(-1)^{n+r+\alpha+k+1} \int_{z \in W_{2}} f(z) \wedge\left[G_{M}\right]_{0, n-k-r-1}(z, \xi)\right. \\
& \left.+(-1)^{\alpha} \int_{z \in \omega_{\tau}} f(z) \wedge\left[R_{M}\right]_{0, n-k-r}(z, \xi)+L_{r} P_{r} f(\xi)\right\} \\
& +(-1)^{n+r+\alpha+1} \int_{z \in W_{2}} \bar{\partial}_{z} f(z) \wedge\left[G_{M}\right]_{0, n-k-r-2}(z, \xi) \\
& +(-1)^{\alpha+k+1} \int_{z \in \omega_{\tau}} \bar{\partial}_{z} f(z) \wedge\left[R_{M}\right]_{0, n-k-r-1}(z, \xi) \\
& +L_{r+1} \bar{\partial}_{\xi} P_{r} f(\xi) \quad \text { pour } 1 \leq r \leq q-2 \text { et } \xi \in \omega^{\prime} .
\end{aligned}
$$

Lemme 3.1. Pour $1 \leq r \leq q-3$, on a

$$
\bar{\partial} P_{r} f(\xi)=P_{r+1} \bar{\partial} f(\xi) .
$$

Démonstration. Soit $f$ une $(n, r)$-forme de classe $C^{1}$ sur $\bar{\omega}_{\tau}$. Alors $\bar{\partial} f$ est une $(n, r+1)$-forme continue sur $\bar{\omega}_{\tau}$. Si $1 \leq r \leq q-3$ alors $1 \leq r+1 \leq q-2$. On a donc, en remplaçant $f$ par $\bar{\partial} f$ dans la formule $(\star \star)$, 


$$
\begin{aligned}
\bar{\partial} f(\xi)= & \bar{\partial}(-1)^{n+r+\alpha^{+}+k} \int_{z \in W_{2}} \bar{\partial} f(z) \wedge\left[G_{M}\right]_{0, n-k-r-2}(z, \xi) \\
& +(-1)^{\alpha^{+}} \bar{\partial} \int_{z \in \omega_{\tau}} \bar{\partial} f(z) \wedge\left[R_{M}\right]_{0, n-k-r-1}(z, \xi)+\bar{\partial} L_{r+1} P_{r+1} \bar{\partial} f(\xi) \\
& +L_{r+2} \bar{\partial} P_{r+1} \bar{\partial} f(\xi) \quad \text { où } \alpha^{+}=\alpha(r+1) .
\end{aligned}
$$

Notons que $\bar{\partial} L_{r+1} P_{r+1} \bar{\partial} f(\xi)+L_{r+2} \bar{\partial} P_{r+1} \bar{\partial} f(\xi)=P_{r+1} \bar{\partial} f(\xi)$. En appliquant $\bar{\partial}$ aux deux membres de $(\star \star)$, on obtient aussi

$$
\begin{aligned}
\bar{\partial} f(\xi)= & \bar{\partial} L_{r+1} \bar{\partial} P_{r} f(\xi) \\
& +(-1)^{n+r+\alpha+1} \bar{\partial}_{\xi} \int_{z \in W_{2}} \bar{\partial}_{z} f(z) \wedge\left[G_{M}\right]_{0, n-k-r-2}(z, \xi) \\
& +(-1)^{\alpha+k+1} \bar{\partial} \int_{z \in \omega_{\tau}} \bar{\partial}_{z} f(z) \wedge\left[R_{M}\right]_{0, n-k-r-1}(z, \xi) .
\end{aligned}
$$

Remarquons qu'à cause de $\bar{\partial}^{2}=0$, on a $\bar{\partial} L_{r+1} \bar{\partial} P_{r} f=\bar{\partial} P_{r} f$. En comparant les expressions obtenues aux deux dernières équations et en remarquant que $\alpha^{+}=\alpha+k+1$, on a alors (après simplification) le résultat annoncé. $\mathrm{Si}$ on tient compte du lemme 3.1 dans la formule $(\star)$, on obtient

$$
\begin{aligned}
f(\xi)= & \bar{\partial}_{\xi}\left\{(-1)^{n+r+\alpha+k+1} \int_{z \in W_{2}} f(z) \wedge\left[G_{M}\right]_{0, n-k-r-1}(z, \xi)\right. \\
& \left.+(-1)^{\alpha} \int_{z \in \omega_{\tau}} f(z) \wedge\left[R_{M}\right]_{0, n-k-r}(z, \xi)+L_{r} P_{r} f(\xi)\right\} \\
& +L_{r+1} P_{r+1} \bar{\partial}_{\xi} f(\xi)+(-1)^{n+r+\alpha+1} \int_{z \in W_{2}} \bar{\partial}_{z} f(z) \wedge\left[G_{M}\right]_{0, n-k-r-2}(z, \xi) \\
& +(-1)^{\alpha+k+1} \int_{z \in \omega_{\tau}} \bar{\partial}_{z} f(z) \wedge\left[R_{M}\right]_{0, n-k-r-1}(z, \xi), \quad \forall \xi \in \omega_{\tau^{\prime}} .
\end{aligned}
$$

Posons

$$
\begin{aligned}
T_{r} f= & (-1)^{n+r+\alpha} \int_{z \in W_{2}} f(z) \wedge\left[G_{M}\right]_{0, n-k-r-1}(z, \cdot) \\
& +(-1)^{\alpha} \int_{z \in \omega_{\tau}} f(z) \wedge\left[R_{M}\right]_{0, n-k-r}(z, \cdot)+L_{r} P_{r} f .
\end{aligned}
$$

On obtient ainsi la formule d'homotopie :

$$
f=\bar{\partial} T_{r} f+T_{r+1} \bar{\partial} f \quad \text { sur } \omega_{\tau^{\prime}}=\omega_{\tau} \cap U_{z_{0}}^{\prime} \text { si } 1 \leq r \leq q-3,
$$

annoncée dans le (i) du théorème 1.1.

Prouvons maintenant la partie (ii) du dit théorème. Pour $r=q-2$ la relation $(\mathcal{R})$ s'écrit

$$
\begin{aligned}
{\left[R_{M}\right]_{0, n-k-q}(z, \xi)=} & (-1)^{k}\left[S_{M}\right]_{0, n-k-q}(z, \xi) \\
& +\left(\bar{\partial}_{z}\left[G_{M}\right]_{0, n-k-q-1}(z, \xi)+\bar{\partial}_{\xi}\left[G_{M}\right]_{0, n-k-q}\right) .
\end{aligned}
$$


Comme $n-k-q<n-k-q+1$, on n'a pas l'annulation de $\left[S_{M}\right]_{0, n-k-q}(z, \xi)$. Donc l'intégrale $\int_{z \in W_{2}} \bar{\partial}_{z} f(z) \wedge\left[S_{M}\right]_{0, n-k-q}(z, \xi)$ n'est pas nécessairement nulle ( $\partial W_{2}$ étant non vide). Par conséquent, la formule $(\star \star)$ n'est pas valable pour $\bar{\partial} f$. Cependant, si $\bar{\partial} f=0$ et $r=q-2$, la formule $(\star \star)$ s'écrit

$$
\begin{aligned}
f(\xi)= & \bar{\partial}_{\xi}\left\{(-1)^{n+r+\alpha+k+1} \int_{z \in W_{2}} f(z) \wedge\left[G_{M}\right]_{0, n-k-r-1}(z, \xi)\right. \\
& \left.+(-1)^{\alpha} \int_{z \in \omega_{\tau}} f(z) \wedge\left[R_{M}\right]_{0, n-k-r}(z, \xi)\right\}+P_{r} f(\xi), \quad \forall \xi \in \omega_{\tau^{\prime}}
\end{aligned}
$$

En appliquant $\bar{\partial}$ aux deux membres de (4), on a $\bar{\partial} P_{r} f(\xi)=0$. Et compte tenu de $(\star)$, on obtient $P_{r} f=\bar{\partial} L_{r} P_{r} f$. Par conséquent, on a

$$
\begin{aligned}
f(\xi)= & \bar{\partial}_{\xi}\left\{(-1)^{n+r+\alpha+k+1} \int_{z \in W_{2}} f(z) \wedge\left[G_{M}\right]_{0, n-k-r-1}(z, \xi)\right. \\
& \left.+(-1)^{\alpha} \int_{z \in \omega_{\tau}} f(z) \wedge\left[R_{M}\right]_{0, n-k-r}(z, \xi)+L_{r} P_{r} f(\xi)\right\}=\bar{\partial} T_{r} f(\xi) .
\end{aligned}
$$

On a donc montré que si $r=q-2$ et $\bar{\partial} f=0$, alors $f=\bar{\partial} T_{r} f$ sur $\omega_{\tau^{\prime}}$.

3.5. Estimation des opérateurs $T_{r}$. Dans la sous-section 3.4 on a construit des opérateurs $T_{r}$ de $C_{n, r}\left(\bar{\omega}_{\tau}\right)$ dans $C_{n, r-1}\left(\omega_{\tau^{\prime}}\right)$. Dans cette partie nous nous proposons de les estimer jusqu'au bord. Plus précisément, nous voulons prouver le théorème suivant :

ThÉORÈme 3.2. Les opérateurs $T_{r}$ obtenus à la fin de la sous-section 3.4 sont des opérateurs continus de $C_{n, r}\left(\bar{\omega}_{\tau}\right)$ dans $C_{n, r-1}^{1 / 2-\varepsilon}\left(\bar{\omega}_{\tau^{\prime}}\right)$ pout tous $\varepsilon>0$ et $1 \leq r \leq q-2$.

Démonstration. La régularité jusqu'au bord de l'intégrale $\int_{z \in W_{2}} f(z) \wedge$ $\left[G_{M}\right]_{0, n-r-k}(z, \cdot)$ a déjà été étudiée dans [6]. Si $f \in C_{n, r}\left(\bar{\omega}_{\tau}\right)$ alors cette intégrale est dans $C^{1 / 2-\varepsilon}\left(\bar{\omega}_{\tau}\right)$ pout tout $0<\varepsilon<1 / 2$. De plus, l'opérateur de Henkin a été estimé dans [5]. Remarques :

(1) Bien que dans [6] les estimations ont été faites dans le cas où $\omega_{\tau}$ est strictement pseudoconvexe, les noyaux $R_{M}$ et $G_{M}$, dans notre travail, ont les mêmes singularités que dans [6]. Les estimations de [6] restent encore valables dans notre cas.

(2) Si $f$ est de classe $C^{\ell}$ sur $\bar{\omega}_{\tau}$, alors, toujours d'après [2], $T_{r} f$ est de classe $C^{1 / 2+\ell} \operatorname{sur} \omega_{\tau}$.

4. Applications. Nous voulons dans cette section retrouver la version $C^{0}$ et dans le cas "concave" de quelques théorèmes d'invariance de cohomologie obtenus dans [5] pour le cas des hypersurfaces réelles. La version $C^{\infty}$ de ces résultats est dans [7]. Nous supposons dans cette section que $M$ est une 
sous-variété $\mathrm{CR}$ générique de $\mathbb{C}^{n}$, de codimension réelle $k$, de classe $C^{3}$ et $q$-concave.

4.1. Notations. Soient $\Omega$ un ouvert de $M$ et $r$ un entier tel que $1 \leq r \leq n$. On note alors $C_{n, r}^{\alpha}(\Omega)$ (resp. $\left.C_{n, r}^{\alpha}(\bar{\Omega})\right)$ l'espace des $(n, r)$-formes différentielles continues sur $\Omega$ (resp. sur $\bar{\Omega}$ ) si $\alpha=0$ et holdériennes d'ordre $\alpha$ si $0<\alpha<1$. On pose $C_{n, r}^{<1 / 2}(\Omega)=\bigcap_{0<\varepsilon<1 / 2} C_{n, r}^{1 / 2-\varepsilon}(\Omega)$ et on définit de même $C_{n, r}^{<1 / 2}(\bar{\Omega})$. On associe à ces espaces les sous-espaces suivants, pour $0 \leq \alpha<1$ :

$$
Z_{n, r}^{\alpha}(\Omega)=C_{n, r}^{\alpha}(\Omega) \cap \operatorname{Ker} \bar{\partial}_{b}, \quad E_{n, r}^{<1 / 2}(\Omega)=Z_{n, r}^{0}(\Omega) \cap \bar{\partial}_{b} C_{n, r-1}^{<1 / 2}(\Omega) .
$$

On définit de même les sous-espaces correspondant à $\bar{\Omega}$. Enfin, on considère les espaces quotients suivants :

$$
H_{<1 / 2}^{n, r}(\Omega)=Z_{n, r}^{0}(\Omega) / E_{n, r}^{<1 / 2}(\Omega) .
$$

On a de même l'espace quotient correspondant à $\bar{\Omega}$.

\subsection{Définitions}

DÉFINITION 4.1. Une configuration affine q-convexe pour $M$ est la donnée d'une collection ordonnée $\left[U, D, \psi, \varrho_{1}, \ldots, \varrho_{k}, \varrho_{-1}, \ldots, \varrho_{-k}\right]$, où :

(1) $U$ est un ouvert convexe de $\mathbb{C}^{n}$,

(2) $\psi, \varrho_{1}, \ldots, \varrho_{k}, \varrho_{-1}, \ldots, \varrho_{-k}$ sont des fonctions à valeurs réelles de classe $C^{2}$ définies sur $U$ telles que :

(a) $D=\{z \in U \mid \psi(z)<0\}$ et $d \psi(z) \neq 0$ pour tout $z \in \partial D$,

(b) pour $I=\left(i_{1}, \cdots, i_{k}\right) \in \mathcal{I}(k)$ un multi-indice (notation voir [2], par exemple), les fonctions $\varrho_{i_{1}}, \ldots, \varrho_{i_{k}}$ sont des fonctions définissantes de $M$ sur $U$,

(c) $\bar{\partial} \varrho_{i_{1}} \wedge \cdots \wedge \bar{\partial} \varrho_{i_{k}} \neq 0$ sur $U$,

(d) si on note $\Omega_{\nu}=\left\{z \in U \mid \varrho_{\nu}(z)<0\right\}, \nu \in\{ \pm 1, \ldots, \pm k\}=J$, alors $M \cap U=\bigcap_{\nu \in J} \bar{\Omega}_{\nu}, U \backslash M=\bigcup_{\nu \in J} \Omega_{\nu}$ et $U=\bigcup_{\nu \in J} \bar{\Omega}_{\nu}$,

(e) pour $1 \leq \ell \leq k$ et $i_{1}, \ldots, i_{\ell} \in J$ tels que $\left|i_{j}\right| \neq\left|i_{k}\right|$ si $j \neq k$, $d \psi(z) \wedge d \varrho_{i_{1}}(z) \wedge \cdots \wedge d \varrho_{i_{\ell}(z)} \neq 0$ sur $\left\{z \in U \mid \psi(z)=\varrho_{i_{1}(z)}=\right.$ $\left.\cdots=\varrho_{i_{\ell}(z)}=0\right\}$

(f) pour tout $\lambda=\left(\lambda_{0}, \lambda_{1}, \ldots, \lambda_{k}\right)$ élément d'un $k$-simplexe, la forme de Levi $\mathcal{L}^{\mathbb{C}^{n}}\left(\lambda_{0} \psi+\lambda_{1} \varrho_{i_{1}}+\cdots+\lambda_{k} \varrho_{i_{k}}\right)$ admet au moins $q+k$ valeurs propres strictement positives en tout point de $U$.

DÉFInITION 4.2. Une bosse q-concave pour $M$ est une collection ordonnée $\left[U_{0}, U_{1}, U_{2}, D_{1}, D_{2}, \psi_{1}, \psi_{2}, \varrho_{1}, \ldots, \varrho_{k}, \varrho_{-1}, \ldots, \varrho_{-k}\right]$ telle que :

(1) $U_{0} \subset \subset U_{1} \subset \subset U_{2}$ sont des ouverts relativement compacts dans $\mathbb{C}^{n}$,

(2) pour $i=1,2, D_{i}=\left\{z \in U_{2} \mid \psi_{i}(z)<0\right\}$, 
(3) pour $i=1,2,\left[U_{2}, U_{2} \backslash \bar{D}_{i},-\psi_{i}, \varrho_{1}, \ldots, \varrho_{k}, \varrho_{-1}, \ldots, \varrho_{-k}\right]$ est une configuration $q$-convexe affine de $M$,

(4) $D_{1} \subseteq D_{2}$ avec $D_{2} \backslash D_{1} \subset \subset U_{0}$.

DÉfinition 4.3. $\left[\theta_{1}, \theta_{2}, V\right]$ est un élément d'extension $q$-concave dans $M$ si $\theta_{1}$ et $\theta_{2}$ sont des ouverts de $M$ à bord $C^{2}$ et $V$ un ouvert relativement compact dans $M$ tels que :

(1) $\theta_{1} \subseteq \theta_{2}$

(2) $\theta_{2} \backslash \theta_{1} \subset \subset V$,

(3) il existe une bosse $q$-concave

$$
\left[U_{0}, U_{1}, U_{2}, D_{1}, D_{2}, \psi_{1}, \psi_{2}, \varrho_{1}, \ldots, \varrho_{k}, \varrho_{-1}, \ldots, \varrho_{-k}\right]
$$

telle que $V=U_{2} \cap M$ et $\theta_{i} \cap V=\left\{z \in V \mid \psi_{i}(z)<0\right\}=D_{i} \cap V$ pour $i=1,2$.

Définition 4.4. On dit que $M$ est $q$-concave à l'infini si :

(1) $M$ est $q$-concave,

(2) il existe une fonction $\varphi$ de classe $C^{2}$ sur $M$, un compact $K$ de $M$ et une constante $C_{\infty} \in \mathbb{R} \cup\{+\infty\}$ tels que :

(a) $\varphi<0$ sur $M \backslash K$,

(b) $\{z \in M \mid \varphi(z) \leq c\}$ est compact pour tout $c<C_{\infty}$,

(c) $\varphi$ est $(q+k)$-concave en tout point de $M \backslash K$.

Lemme 4.1. Soit $\left[\theta_{1}, \theta_{2}, V\right]$ un élément d'extension $q$-concave dans $M$. Alors l'application restriction $H_{<1 / 2}^{n, r}\left(\bar{\theta}_{2}\right) \rightarrow H_{<1 / 2}^{n, r}\left(\bar{\theta}_{1}\right)$ est un isomorphisme si $0 \leq r \leq q-2$.

Démonstration. Soient $\left[\theta_{1}, \theta_{2}, V\right]$ un élément d'extension $q$-concave dans $M$, et $\left[U_{0}, U_{1}, U_{2}, D_{1}, D_{2}, \psi_{1}, \psi_{2}, \varrho_{1}, \ldots, \varrho_{k}, \varrho_{-1}, \ldots, \varrho_{-k}\right]$ une bosse $q-$ concave associée à cet élément. Soit $r \in \mathbb{N}$ tel que $0 \leq r \leq q-1$.

Supposons $2 \leq r \leq q-2$. Montrons que l'application restriction définie ci-dessus est surjective. Soit $f_{1} \in C_{n, r}^{<1 / 2}\left(\bar{\theta}_{1}\right)$ telle que $\bar{\partial}_{b} f_{1}=0$ sur $\theta_{1}$. On doit trouver $f_{2} \in C_{n, r}^{<1 / 2}\left(\bar{\theta}_{2}\right)$ telle que $f_{1}-f_{2} \in E_{n, r}^{<1 / 2}\left(\bar{\theta}_{1}\right)$. Comme $\bar{\partial}_{b} f_{1}=0$, d'après la formule d'homotopie du théorème 1.1 établie dans la section 3 , on sait résoudre le $\bar{\partial}_{b}$ sur $\omega_{\tau_{i}}=D_{i} \cap U_{2} \cap M, i=1,2$. Par conséquent il existe $u=T_{r} f_{1} \in C_{n, r-1}^{<1 / 2}\left(\overline{D_{1} \cap U_{1} \cap M}\right)$ tel que $\bar{\partial}_{b} u=f_{1}$ sur $D_{1} \cap U_{2} \cap M=$ $D_{1} \cap V=\theta_{1} \cap V \subset \theta_{1}$. Soit alors $\chi \in C^{\infty}(M)$ tel que $\chi \equiv 1$ sur $\theta_{2} \backslash \theta_{1}$ et supp $\chi \subset \subset V$. Posons $u_{1}=\chi T_{r} f_{1}$ sur $\theta_{1}$ et

$$
f_{2}= \begin{cases}0 & \operatorname{sur} \bar{\theta}_{2} \backslash \theta_{1}, \\ f_{1}-\bar{\partial}_{b}\left(\chi T_{r} f_{1}\right) & \text { sur } \bar{\theta}_{1} .\end{cases}
$$

On a donc $u_{1} \in C_{n, r-1}^{<1 / 2}\left(\bar{\theta}_{1}\right)$ et $f_{2} \in C_{n, r}^{<1 / 2}\left(\bar{\theta}_{2}\right)$ avec $f_{1}-f_{2}=\bar{\partial}_{b} u_{1}$. 
Montrons que l'application restriction est aussi injective. Soit $f_{2} \in Z_{n, r}^{0}\left(\bar{\theta}_{2}\right)$ avec $2 \leq r \leq q-2$ et telle qu'il existe $u_{1} \in C_{n, r}^{<1 / 2}\left(\bar{\theta}_{1}\right)$ avec $\bar{\partial}_{b} u_{1}=f_{2}$ sur $\theta_{1}$. Comme $\bar{\partial}_{b} f_{2}=0$ sur $\theta_{2}$, d'après notre formule d'homotopie il existe $v=T_{r} f_{2} \in C_{n, r-1}^{<1 / 2}\left(\overline{\theta_{2} \cap U_{2}}\right)$ telle que $\bar{\partial}_{b} v=f_{2}$ sur $\theta_{2} \cap U_{1}$. De plus, sur $U_{2} \cap \theta_{1} \subset U_{2} \cap \theta_{2}$, on a $\bar{\partial}_{b}\left(v-u_{1}\right)=0$. Par suite, $v-u_{1} \in C_{n, r-1}^{<1 / 2}\left(\overline{\theta_{2} \cap U_{1}}\right)$ implique $v-u_{1} \in Z_{n, r-1}^{0}\left(\overline{\theta_{1} \cap U_{2}}\right)$. Comme $2 \leq r \leq q-2$, on a $1 \leq r-1 \leq$ $q-3$. La même formule d'homotopie nous permet de trouver $w=T_{r-1}(v-u)$ avec $w \in C_{n, r-2}^{<1 / 2}\left(\overline{\theta_{1} \cap U_{0}}\right)$ et telle que $\bar{\partial}_{b} w=v-u_{1}$. Si $\chi \in C^{\infty}(M)$ avec $\chi \equiv 1$ sur $\overline{\theta_{2} \backslash \theta_{1}}$ et supp $\chi \subset \subset V$, alors prenons $u_{2}=u_{1}-\bar{\partial}_{b}(\chi w) \operatorname{sur} \theta_{2}$. On a $u_{2} \in C_{n, r-1}^{<1 / 2}\left(\bar{\theta}_{2}\right)$ avec $\bar{\partial}_{b} u_{2}=f_{2} \operatorname{sur} \bar{\theta}_{2}$.

Supposons $r=1$. On utilise alors $v-u_{1}=w$ où $w$ est une $(n, 0)$-forme CR sur $\overline{U_{1} \cap \theta_{1}}$, et on pose alors $u_{2}=u_{1} \operatorname{sur} \bar{\theta}_{1}$ et $u_{2}=v+w \operatorname{sur} \theta_{2} \backslash \theta_{1}$.

Enfin, supposons $r=0$. L'injectivité provient alors du fait que dans les variétés $\mathrm{CR}$ 1-concaves le principe du prolongement analytique est valable pour les formes $\mathrm{CR}$. Ainsi une $(n, 0)$-forme $\mathrm{CR}$ sur $\bar{\theta}_{2}$ qui s'annule sur $\bar{\theta}_{1}$ est nécessairement nulle sur $\theta_{2}$. La surjectivité découle du fait suivant : si $f$ est une $(n, 0)$-forme sur $\bar{\theta}_{1}$, elle est CR sur $\overline{U_{1} \cap \theta_{1} \cap D_{i}} \subset \bar{\theta}_{1}$, donc admet une extension CR sur $M \cap U_{0}$ et donc sur $\bar{\theta}_{2}$ (cf. thm. 7.2.1 de [1]).

Remarquons que pour $r=q-1$, on a le lemme suivant :

Lemme 4.2. Soient $M$ une sous-variété $C R$ générique q-concave de $\mathbb{C}^{n}$, de classe $C^{3}$ et de codimension $k$. Soit $\left[\theta_{1}, \theta_{2}, V\right]$ un élément d'extension q-concave dans $M$ tel que $V \subseteq B(\xi, R) \cap M$, où $\xi$ est un point de $\overline{\theta_{2} \backslash \theta_{1}}$. Soit $f \in C_{n, q-1}^{<1 / 2}(M)$ avec $\bar{\partial}_{b} f=0$. Alors pour tout voisinage $W$ dans $M$ de $\overline{\theta_{2} \backslash \theta_{1}}$, il existe $u_{2} \in C_{n, q-2}^{<1 / 2}\left(\bar{\theta}_{2}\right)$ avec $\bar{\partial}_{b} u_{2}=f$ sur $\theta_{2}$ et $u_{2}=u_{1}$ sur $\bar{\theta}_{1} \backslash W$.

Démonstration. On remplace le théorème 2.3.2(i) de [7] par la formule d'homotopie établie dans [2]. La preuve est alors identique à celle de [7].

Lemme 4.3. Soit $[D, G]$ une extension strictement $q$-concave dans $M$ et $\left(U_{i}\right)_{i \in I}$ un recouvrement de $\overline{G \backslash D}$ par des ouverts de $M$. Alors il existe des ouverts de $M: \theta_{0}, \ldots, \theta_{N}, V_{0}, \ldots, V_{N-1}$ tels que:

(1) $\theta_{0}=D$ et $\theta_{N}=G$,

(2) pour tout $i \in\{0, \ldots, N-1\},\left[\theta_{i}, \theta_{i+1}, V_{i}\right]$ est un élément d'extension $q$-concave dans $M$,

(3) pour tout $i \in\{0, \ldots, N-1\}$, il existe $j_{0} \in I$ tel que $V_{i} \subset \subset U_{j_{0}}$.

On pourra se reporter à [7] pour la définition de la notion d'extension strictement $q$-concave et la preuve du lemme 4.3.

On déduit des lemmes 4.1 et 4.2 le théorème suivant : 
THÉORÈme 4.1. Soit $[D, G]$ une extension strictement $q$-concave dans $M$. Alors l'application restriction : $H_{<1 / 2}^{n, r}(\bar{G}) \rightarrow H_{<1 / 2}^{n, r}(\bar{D})$ est un isomorphisme si $0 \leq r \leq q-2$.

On a aussi le résultat suivant :

ThÉORÈme 4.2. Soit $M$ une sous-variété $C R$ générique de $\mathbb{C}^{n}$, de classe $C^{3}$ et de codimension réelle $k$. On suppose que $M$ est q-concave à l'infini. Soit $D$ un domaine de $M$ tel que $D=K \cup\{z \in M \backslash K \mid \varphi(z)<0\}$ où $K \subset \subset M$. Alors l'application restriction $H_{<1 / 2}^{n, r}(M) \rightarrow H_{<1 / 2}^{n, r}(\bar{D})$ est un isomorphisme pour tout $r$ tel que $0 \leq r \leq q-2$, et donc $\operatorname{dim}_{\mathbb{C}} H^{n, r}(M)<+\infty$ pour un tel $r$. De plus, si $r=q-1$, alors elle est injective.

Démonstration. Soient $K, \varphi$ comme dans la définition 4.4. Définissons $D$ comme dans l'énoncé du théorème 4.2. Alors, compte tenu de la formule d'homotopie du théorème 1.1, le reste de la preuve est identique à celle de la proposition 3.1 , section 3 , chapitre 7 de [4].

\section{Références}

[1] R. A. Airapetjan and G. M. Henkin, Integral representations of differential forms on Cauchy-Riemann manifolds and the theory of CR-functions, Russian Math. Surveys 39 (1984), no. 3, 41-118.

[2] M. Y. Barkatou et Ch. Laurent-Thiébaut, Solutions fondamentales et estimations optimales pour l'opérateur de Cauchy-Riemann tangentiel, Michigan Math. J. 54 (2006), 545-586.

[3] G. M. Henkin and J. Leiterer, Theory of Functions on Complex Manifolds, Monogr. Math. 79, Birkhäuser, 1984.

[4] Ch. Laurent-Thiébaut, Théorie des fonctions holomorphes de plusieurs variables, InterEditions / CNRS Editions, 1997.

[5] Ch. Laurent-Thiébaut and J. Leiterer, Andreotti-Grauert Theory on Real Hypersurfaces, Quaderni, Scuola Normale, Pisa, 1995.

[6] Ch. Laurent-Thiébaut and M. C. Shaw, Boundary Hölder and $L^{p}$ estimates for local solutions of the tangential Cauchy-Riemann equation, Trans. Amer. Math. Soc. 357 (2005), 151-177.

[7] H. Ricard, Résolution avec régularité jusqu'au bord de l'équation de Cauchy-Riemann dans des domaines à coins et de l'équation de Cauchy-Riemann tangentielle en codimension quelconque, thèse, Univ. de Grenoble 1, 2002.

[8] S. Sambou, Théorème de séparation de type Andreotti-Vesentini sur les variétés CR génériques, Ann. Mat. 185 (2006), 381-394.

Département de Mathématiques et Informatique

Faculté des Sciences et Techniques

Université Cheikh Anta Diop de Dakar

Dakar, Sénégal

E-mail: tourebocar@yahoo.fr, ssambou@refer.sn

Received 10.7.2006

and in final form 21.2.2007 